\title{
COMETAS: I - POPULAÇÕES E INTENSIDADES DE NOVE BANDAS VIBRACIONAIS DO MONOSSULFETO DE CARBONO $\left({ }^{12} \mathrm{C}^{32} \mathrm{~S}\right)$
}

\author{
MARCUS VINÍCIUS CANAVES ${ }^{1}$ \\ GILBERTO CARLOS SANZOVO ${ }^{2}$
}

CANAVES, M. V; SANZOVO, G. C. Cometas: I - Populações e Intensidades de Nove Bandas Vibracionais do Monossulfeto de Carbono $\left({ }^{12} \mathrm{C}^{32} \mathrm{~S}\right)$. Semina:Ci. Exatas/Tecnol., Londrina, v. 17, n. 4, p. 369-375, dez. 1996.

RESUMO: Neste trabalho nós realizamos um estudo teórico do mecanismo de excitação por fluorescência ressonante, para a espécie molecular monossulfeto de carbono $C^{12} C^{32} S$ ), nas comas dos Cometas West (1976VI) e P/Bradfield (1979X), através do campo de radiação solar o que nos permitiu deduzir as populações relativas e as intensidades de 9 bandas vibracionais da molécula.

PALAVRAS-CHAVE: Cometas; fluorescência ressonante; CS: populações vibracionais; CS: intensidades de bandas vibracionais.

\section{INTRODUÇÃO}

Parece haver consenso, por parte dos Astrônomos, que os cometas conservaram intacta a maior parte das características físicas e químicas reinantes no material primitivo da nuvem interestelar que formou o Sol e os Planetas. No reservatório de Oort, os núcleos cometários encontram-se fracamente ligados ao Sol por forças gravitacionais. Desse modo, em decorrência de flutuações do campo gravitacional no qual nosso sistema solar se encontra imerso, alguns desses núcleos cometários eventualmente podem ser capturados pela influência massiva de Júpiter (Lüsi, 1981). Já no interior do Sistema Solar, quando a distância heliocêntrica for inferior a 3,0 UA, o campo de radiação do Sol atuará no sentido de sublimar, em parte, o material contido no núcleo formando a coma, na qual as espécies cometárias serão sintetizadas através de reações químicas como a fotoionização e a fotodissociação.

O espectro eletromagnético produzido pelo Sol é bem conhecido (Labs e Neckel, 1968; Arvesen et al., 1969; Broadfoot, 1972). Neste trabalho, nós realizamos um estudo teórico com a aplicação do mecanismo de fluorescência ressonante à espécie molecular monossulfeto de carbono $\left({ }^{12} \mathrm{C}^{32} \mathrm{~S}\right)$ para as comas dos Cometas West (1976VI) e P/Bradfield (1979X), através da ação do campo de radiação solar. O mecanismo de excitação por fluorescência ressonante é muito mais eficiente para a excitação das emissões cometárias do que o processo de excitação por colisões da radiação com átomos, íons ou moléculas (Krishna Swamy, 1981). As emissões com $1=2576,2663$ e 2507 Ang. foram inicialmente observadas nos espectros ultravioletas (UV) do Cometa West por Smith et ai (1980) e identificadas como pertencentes ao CS, a primeira molécula cometeria detectada que contém o enxofre. Na seção 2 nós descrevemos, inicialmente, o mecanismo de fluorescência ressonante, responsável direto pelas emissões das espécies cometárias (Krishna Swamy, 1981) e, em seqüência, verificamos a estrutura vibracional do CS e revemos a teoria que nos permitiu deduzir as populações fracionais e as intensidades das bandas vibracionais. Na seção 3 nós comparamos nossos resultados teóricos com os dados observacionais e teóricos, disponíveis na Literatura. Finalmente, na seção 4, nós apresentamos a discussão dos resultados obtidos.

1 -Aluno de Graduação em Física e de Iniciação Científica

2- Docente do Departamento de Física da Universidade Estadual de Londrina - Caixa Postal 6001, Londrina, PR, CEP 86051 -990 Endereço Eletrônico: gsanzovo@uel.br 


\section{ASPECTOS TEÓRICOS}

\subsubsection{Mecanismo de Excitação por Fluorescência Ressonante}

Esse mecanismo será explicado aqui, de modo simples, considerando uma espécie molecular $\mathrm{M}$ e 3 níveis de energia. A absorção da radiação solar com energia hv aumenta a população da molécula no nível excitado (não meta-estável), $X_{3}$. Como o tempo de vida radiativo em $\mathrm{X}_{3}$ é, normalmente, pequeno ( $\mathrm{t}_{\mathrm{x}_{3}}$ $10^{-9} \mathrm{~s}$ ), a molécula poderá decair para os níveis inferiores $X_{2}$, também excitado, eX $X_{1}$ (fundamental), com as conseqüentes emissões de fótons com energias $h v_{2}$ e hv $v_{1}$ gerando as linhas subordinada e de ressonância, respectivamente. Na Figura 1 nós esquematizamos a situação apresentada. Se a espécie molecular não encontrar outro fóton ou parceiro coiisional no (limitado) intervalo de tempo correspondente ao tempo de vida de $X_{2}$, ela poderá decair até o nível fundamental, com a conseqüente emissão de um fóton com energia $\mathrm{hv}_{3}$. Os cometas absorvem, geralmente, fótons solares com energia hv por fluorescência ressonante e reemitem radiação com energia $h v_{1}$.

O Sol emite um espectro eletromagnético que varia desde o ultravioleta (UV) até o infravermelho (IV) distante, com a superposição das linhas de Fraunhoffer devido à presença de espécies atômicas e moleculares em sua fotosfera. Deste modo, ele representa um excelente campo de radiação estelar para a excitação, por fluorescência ressonante, de espécies cometárias atômicas e moleculares. Em um cometa, se a emissão da radiação solar com energia hv for coincidente com aquela correspondente de uma linha da espécie molecular (ou atômica) com energia equivalente a $E_{x_{3}}-E_{x_{1}}$, a espécie será excitada até o nível molecular $X_{3}$ e, como tx3 é pequeno, ela irá decair até $X_{1}$, provocando a emissão da linha de ressonância com energia $h_{1}$. Se a diferença de energia $\mathrm{DE}\left(\mathrm{X}_{3}-\mathrm{X}_{1}\right)$ for coincidente com uma linha de absorção de Fraunhoffer, a linha de emissão com energia hv, não aparecerá no espectro do cometa. O efeito Doppler aparece como conseqüência da velocidade radial do cometa com relação ao Sol, o que complica o mecanismo de excitação. As conseqüências do desvio Doppler foram inicialmente averiguadas nas linhas rotacionais da banda vibracional $(0,0)$ do radical $C N$ por Swings (1941) e, por isso, seu efeito sobre as intensidades das linhas é, atualmente, conhecido por Efeito Swings.

\subsection{A Estrutura Vibracional do Monossulfeto de Carbono}

A estrutura vibracional do monossulfeto de carbono foi investigada de acordo com o modelo do oscilador anharmônico onde o potencial é representado pela função de Morse, dada por (Herzberg, 1950)

$$
V=D_{0}\left[1-\exp \left[\beta\left(r-r_{e}\right)\right]\right\}^{2}
$$

onde $D$ é a energia de dissociação da molécula e b é a constante de Morse, que leva em consideração sua massa reduzida. O termo vibracional (Herzberg, 1950) é obtido substituindo-se (1) na equação de Schródinger, o que resulta em

$$
G(v)=w_{c}\left(v+\frac{1}{2}\right)-w_{c} x_{c}\left(v+\frac{1}{2}\right)^{2}+w_{c} y_{0}\left(v+\frac{1}{2}\right)^{e}-\ldots
$$

em que $v$ é o número quântico vibracional, $w_{e}$ é conhecido como freqüência de equilíbrio da molécula, $e_{e}$ e $y_{e}$ são as constantes de anharmonicidade.

De acordo com Herzberg (1950), a energia interna de uma molécula diatômica é a soma das energias eletrônica $\left(E_{e}\right)$, vibracional $\left(E_{v}\right)$ e rotacional $\left(E_{r}\right)$. Deste modo, o número de ondas $\left(e \mathrm{~m} \mathrm{~cm}^{1}\right.$ ) da radiação emitida (ou absorvida) em uma transição rotovibracional envolvendo os estados eletrônicos $\mathrm{A}^{1} \Pi$ e $X^{1} \Sigma^{+}$, vale

$$
\sigma=\left(T_{a}-T_{e}\right)+\left[G\left(v^{\prime}\right)-G\left(v^{\prime \prime}\right)\right]+\left[F_{r}\left(d^{\prime}\right)-F_{e}\left(J^{\prime \prime}\right)\right]
$$

ou

$$
\omega=\omega_{u}+\omega_{v}+\omega_{r}
$$

Em (3), o termo vibracional corresponde à equaçăo (2) enquanto que o termo rotacional é pequeno quando comparado $\operatorname{com} \sigma_{e}$ e $\sigma_{v}$. Assim, se substituirmos (2) em (3) e, se nos limitarmos ao termo quadrático, nós teremos que

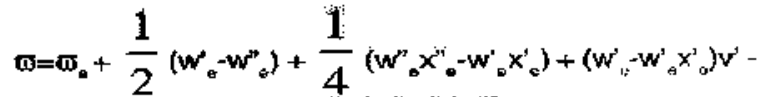

$$
\begin{aligned}
& \left(w_{0}^{\prime} x_{0}^{\prime}\right) v^{2} 2-\left(w_{0}^{\prime \prime}-w^{\prime \prime} x^{\prime \prime}{ }_{0}\right) v^{\prime \prime}+\left(w_{0}{ }_{0} x^{\prime \prime}{ }_{0}\right) v^{\prime 2}+\ldots
\end{aligned}
$$

onde $v$ ' $e v^{\prime \prime}$ são os números quânticos dos estados eletrônicos $\mathrm{A}^{1} \Pi$ e $\mathrm{X}^{1} \Sigma^{+}$, respectivamente. Se nós definirmos

$$
\omega_{\infty}=\omega\left(v^{\prime}=0 \rightarrow v^{\prime \prime}=0\right)=\omega_{0}+\frac{1}{2}\left(w_{0}^{\prime}-w_{e}^{\prime \prime}\right)+\frac{1}{4}\left(w_{a}^{\prime \prime} x_{c}^{\prime \prime}-w_{0}^{\prime} x_{0}\right)
$$

como sendo a freqüência (em $\mathrm{cm}^{-1}$ ) da banda vibracional $(0,0)$ para o sistema eletrônico considerado, a freqüência de uma banda vibracional genérica $\left(v^{\prime}, v^{\prime \prime}\right)$ valerá 


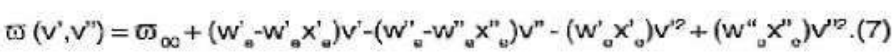

Como não há qualquer regra de seleção para o número quântico vibracional v, a equação (7) fornecerá, para a molécula CS, todas as possíveis transições entre os diferentes níveis vibracionais, nos estados eletrônicos $\mathrm{A}^{1} \Pi$ e $X^{1} \Sigma^{*}$.

\subsection{As Intensidades das Bandas Vibracionais do CS em Cometas}

Para calcular as intensidades das bandas vibracionais do monossulfeto de carbono em cometas através do mecanismo de fiuorescência ressonante, nós procedemos de forma semelhante a Krishna Swamy (1981). Desta maneira, as populações relativas a cada $n$ ível vibracional foram obtidas a partir de um sistema de equações simultâneas, dado por

$$
n_{i} \sum_{k=6}^{11}\left[B_{k k} \rho v_{i k}\right]=\sum_{k=6}^{N 1} n_{k}\left[B_{k} \rho v_{k j}+A_{k}\right]
$$

com $i=0,1, \ldots .5$, para os niveis vibracionais do estado eletrônico fundamental $X^{1} \Sigma^{+}$, e

$$
n_{1} \sum_{i=0}^{5}\left[\mathrm{~B}_{11} \rho v_{11}+\mathrm{A}_{\mathrm{i}}\right]=\sum_{i=0}^{5}\left[\mathrm{n}_{i} \mathrm{~B}_{n} \rho v_{\mathrm{l}}\right]
$$

com $j=6,7, \ldots, 11$, para os níveis vibracionais do estado eletrônico superior. Nestas equaçōes, $n$ representa a fraçāo de moléculas em cada nível enquanto que $\rho v$ (em erg.cm ${ }^{2}$ ) é a densidade da radiação solar na freqüência da transiçāo considerada. Os coeficientes de Einstein A e $B$ relacionam-se com as probabilidades de emissão espontânea e de absorçāo, respectivamente. Nós utilizamos a condiçăo de normalização

$$
\sum_{i=0}^{11} n_{t}=1
$$

visto ser o sistema constituído por $(n-1)$ equações linearmente independentes. As equações (8) e (9) representam o resultado do balanço radiativo entre as transições que povoam e aquelas que despovoam cada um dos 12 níveis vibracionais considerados.
As probabilidades de emissão espontânea foram obtidas da expressão de Larsson (1983)

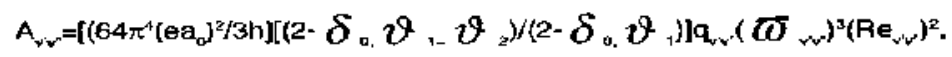

onde $\left[64 \pi^{4}\left(e a_{0}\right)^{2 / 3 h}\right]=2,026 \times 10^{-6},\left[\left(2-\delta_{0} \vartheta_{1+} \vartheta_{2}\right) /(2-\right.$ $\left.\left.S_{0}, \vartheta_{1}\right)\right]=2$ para a transiçāo eletrônica considerada $e, q_{y^{\prime} v^{m}}$ são os fatores de Franck-Condon para as bandas do sistema eletrônico (A-X) da molécula, vinculados à regra da soma vibracional

$$
\sum_{r} a_{r v}=\Sigma_{r} q_{v v}=1
$$

Em (11), $\boldsymbol{\sigma}_{\gamma^{*}}$ representam as freqüências (em $\mathrm{cm}^{-1}$ ) das bandas vibracionais do Sistema (A-X), e $\mathrm{Re}_{y^{\prime} y^{\prime \prime}}$ é o momento da transição eletrônica (em unidades de ea ) para o referido sistema.

\section{RESULTADOS OBTIDOS}

Na Tabela 1 nós apresentamos os parâmetros relativos a 36 bandas vibracionais da molécula monossulfeto de carbono. Os valores de $q_{v^{\prime} v^{\prime}}$ e $R e_{v^{\prime} v^{\prime}}$ foram obtidos de Coxon et al (1976). As probabilidades de emissão espontânea (em s$~^{-1}$ ) foram deduzidas a partir de (11) enquanto que os coeficientes de absorção foram determinados a partir da expressão $(1,52)$ de Herzberg (1950). Dessa maneira, nós obtivemos as populaçōes relativas a 12 níveis vibracionais envolvendo os estados eletrônicos do Sistema (A-X). Como a molécula é heteronuclear nós consideramos, também, as transições puramente vibracionais para o estado eletrônico fundamental $X^{1} \Sigma^{*}$. Essas transiçŏes se situam no infravermelho, e os resultados que obtivemos aparecem mostrados na Tabela 2. As probabilidades de emissảo espontanea foram deduzidas a partir da equação (795) de Penner (1959), onde consideramos $|\mu|=$ $1,025913 \mathrm{D} / \mathrm{Ang}$. como sendo a coeficiente do termo linear da expressão do momento dipolar da molécula. Os fluxos solares, no ultravioleta, para os comprimentos de onda entre 2100 e 3200 Ang., foram obtidos de Broadfoot (1972) e, para as transiçóes no infravermeiho, entre 2 e $8 \mu$, de Allen (1973). 
TABELA 1. Origens das bandas vibracionais da molecula ${ }^{12} \mathrm{C}^{32} \mathrm{~S}$

\begin{tabular}{|c|c|c|c|c|c|c|}
\hline $\begin{array}{c}\text { BANDA } \\
\text { VIBRAC. } \\
\text { [v',v"] }\end{array}$ & $\begin{array}{c}\text { COMPRIM. } \\
\text { DE ONDA } \\
\boldsymbol{\lambda} \\
{[\hat{\mathbf{A}}]}\end{array}$ & $\begin{array}{c}\text { NÚMERO } \\
\text { DE ONDA } \\
\boldsymbol{0} \\
{\left[\mathrm{cm}^{-1}\right]}\end{array}$ & $q_{\mathbf{v}^{\prime}, \mathbf{w}^{\prime \prime}}$ & $\left(R e_{v^{\prime} . v^{m}}\right)^{2}$ & $\begin{array}{c}\text { TAXA EMIS. } \\
\text { ESPONT. } \\
\text { A }_{v^{\prime}, v^{\prime \prime}} \\
{\left[\mathbf{s}^{-1}\right]}\end{array}$ & $\begin{array}{c}\text { COEFIC. DE } \\
\text { ABSORÇĀO } \\
\text { B }_{v^{\prime}, v^{\prime \prime}} \\
{\left[\mathbf{c m}^{2} \mathbf{e r g}^{-1} \mathbf{s}^{-1}\right]}\end{array}$ \\
\hline$(0,0)$ & 2575,952 & $38,821 \times 10^{3}$ & $0,83 \times 10^{\circ}$ & 0,101 & $1,987 \times 10^{7}$ & $6,803 \times 10^{7}$ \\
\hline$(0,1)$ & 2662,697 & $37,556 \times 10^{3}$ & $0,151 \times 10^{0}$ & 0,080 & $2,593 \times 10^{6}$ & $9,805 \times 10^{6}$ \\
\hline$(0,2)$ & 2755,326 & $36,293 \times 10^{3}$ & $0,160 \times 10^{-1}$ & 0,067 & $2,076 \times 10^{5}$ & $8,698 \times 10^{5}$ \\
\hline$(0,3)$ & 2852,749 & $35,053 \times 10^{3}$ & $0,153 \times 10^{-2}$ & 0,058 & $1,549 \times 10^{4}$ & $7,203 \times 10^{4}$ \\
\hline$(0,4)$ & 2955,742 & $33,832 \times 10^{3}$ & $0,200 \times 10^{-3}$ & 0,060 & $1,883 \times 10^{3}$ & $9,740 \times 10^{3}$ \\
\hline$(0, \overline{5})$ & 3064,582 & $32,631 \times 10^{3}$ & $0,460 \times 10^{-5}$ & 0,023 & $1,489 \times 10^{1}$ & $8,584 \times 10^{1}$ \\
\hline$(1,0)$ & 2508,148 & $39,870 \times 10^{3}$ & $0,152 \times 10^{0}$ & 0,120 & $4,684 \times 10^{6}$ & $1,480 \times 10^{7}$ \\
\hline$(1,1)$ & 2589,966 & $38,610 \times 10^{3}$ & $0,513 \times 10^{\circ}$ & 0,099 & $1,187 \times 10^{7}$ & $4,131 \times 10^{7}$ \\
\hline$(1,2)$ & 2677,716 & $37,345 \times 10^{3}$ & $0,268 \times 10^{0}$ & 0,079 & $4,468 \times 10^{6}$ & $1,718 \times 10^{7}$ \\
\hline$(1,3)$ & 2769,357 & $36,109 x+0^{3}$ & $0,574 \times 10^{-1}$ & 0,067 & $7,337 \times 10^{5}$ & $3,137 \times 10^{6}$ \\
\hline$(1,4)$ & 2866,799 & $34,882 \times 10^{3}$ & $0,740 \times 10^{-2}$ & 0,056 & $7,127 \times 10^{1}$ & $3,363 \times 10^{5}$ \\
\hline$(1,5)$ & 2970,831 & $33,661 \times 10^{3}$ & $0,983 \times 10^{-3}$ & 0,053 & $8,052 \times 10^{3}$ & $4,229 \times 10^{4}$ \\
\hline$(2,0)$ & 2444,293 & $40,912 \times 10^{3}$ & $0,159 \times 10^{-1}$ & 0,145 & $6,397 \times 10^{5}$ & $1,871 \times 10^{6}$ \\
\hline$(2,1)$ & 2523,158 & $39,633 \times 10^{3}$ & $2,283 \times 10^{0}$ & 0,115 & $8,210 \times 10^{6}$ & $2,641 \times 10^{7}$ \\
\hline$(2,2)$ & 2605,979 & $38,373 \times 10^{3}$ & $0,261 \times 10^{\circ}$ & 0,098 & $5,856 \times 10^{6}$ & $2,076 \times 10^{7}$ \\
\hline$(2,3)$ & 2692,736 & $37,137 \times 10^{3}$ & $0,306 \times 10^{0}$ & 0,077 & $4,890 \times 10^{6}$ & $1,912 \times 10^{7}$ \\
\hline$(2,4)$ & 2785,384 & $35,902 \times 10^{3}$ & $0,109 \times 10^{2}$ & 0,066 & $1,349 \times 10^{6}$ & $5,839 \times 10^{6}$ \\
\hline$(2,5)$ & 2882,844 & $34,688 \times 10^{3}$ & $0,212 \times 10^{-1}$ & 0,056 & $2,008 \times 10^{5}$ & $9,636 \times 10^{5}$ \\
\hline$(3,0)$ & 2385,403 & $41,922 \times 10^{3}$ & $0,111 \times 10^{-2}$ & 0,179 & $5,932 \times 10^{4}$ & $1,613 \times 10^{5}$ \\
\hline$(3,1)$ & 2460,300 & $40,645 \times 10^{3}$ & $0,481 \times 10^{-1}$ & 0,139 & $1,819 \times 10^{6}$ & $5,426 \times 10^{6}$ \\
\hline$(3,2)$ & 2538,168 & $39,398 \times 10^{3}$ & $0,350 \times 10^{0}$ & 0,112 & $9,714 \times 10^{6}$ & $3,182 \times 10^{7}$ \\
\hline$(3,3)$ & 2661.961 & $37,566 \times 10^{3}$ & $0,978 \times 10^{-1}$ & 0,099 & $2,080 \times 10^{6}$ & $7,859 \times 10^{6}$ \\
\hline$(3,4)$ & 2708,754 & $36,917 \times 10^{3}$ & $0,292 \times 10^{0}$ & 0,075 & $4,465 \times 10^{5}$ & $1,777 \times 10^{7}$ \\
\hline$(3,5)$ & 2801,412 & $35,696 \times 10^{3}$ & $0,157 \times 10^{0}$ & 0,064 & $1,852 \times 10^{5}$ & $8,156 \times 10^{6}$ \\
\hline$(4,0)$ & 2330,489 & $42,909 \times 10^{3}$ & $0,284 \times 10^{-4}$ & 0,266 & $2,418 \times 10^{3}$ & $6,130 \times 10^{3}$ \\
\hline$(4,1)$ & 2401,408 & $41,642 \times 10^{3}$ & $0,361 \times 10^{-2}$ & 0,182 & $1,922 \times 10^{5}$ & $5,331 \times 10^{5}$ \\
\hline$(4,2)$ & 2476,306 & $40,383 \times 10^{3}$ & $0,926 \times 10^{-1}$ & 0,133 & $3,286 \times 10^{5}$ & $9,994 \times 10^{6}$ \\
\hline$(4,3)$ & 2555,176 & $39,136 \times 10^{3}$ & $0,352 \times 10^{0}$ & 0,108 & $9,233 \times 10^{5}$ & $3,085 \times 10^{7}$ \\
\hline$(4,4)$ & 2638,005 & $37,907 \times 10^{3}$ & $0,126 \times 10^{-1}$ & 0,112 & $3,115 \times 10^{5}$ & $1,145 \times 10^{6}$ \\
\hline$(4,5)$ & 2725,771 & $36,687 \times 10^{3}$ & $0,242 \times 10^{\circ}$ & 0,073 & $3,535 \times 10^{6}$ & $1,434 \times 10^{7}$ \\
\hline$(5,0)$ & 2278,558 & $43,887 \times 10^{3}$ & $0,552 \times 10^{-5}$ & 0,053 & $1,010 \times 10^{2}$ & $2,385 \times 10^{2}$ \\
\hline$(5,1)$ & 2346,493 & $42,617 \times 10^{3}$ & $0,814 \times 10^{-4}$ & 0,348 & $8,881 \times 10^{3}$ & $2,298 \times 10^{4}$ \\
\hline$(5,2)$ & 2418,413 & $41,349 \times 10^{3}$ & $0,111 \times 10^{-1}$ & 0,169 & $5,383 \times 10^{5}$ & $1,525 \times 10^{6}$ \\
\hline$(5,3)$ & 2493,313 & $40,107 \times 10^{3}$ & $0,152 \times 10^{\circ}$ & 0,127 & $5,041 \times 10^{6}$ & $1,565 \times 10^{7}$ \\
\hline$(5,4)$ & 2572,184 & $38,878 \times 10^{3}$ & $0,293 \times 10^{0}$ & 0,105 & $7,340 \times 10^{6}$ & $2,502 \times 10^{7}$ \\
\hline$(5,5)$ & 2656,016 & $37,650 \times 10^{3}$ & $0,633 \times 10^{-2}$ & 0,057 & $7,839 \times 10^{4}$ & $2,942 \times 10^{5}$ \\
\hline
\end{tabular}

Os números de onda vibracionais foram obtidos das constantes $w_{*}^{\prime}=1073,4 \mathrm{~cm}^{-1}$ e $w^{\prime} x^{\prime}=10,1 \mathrm{~cm}^{-1}$, para o estado eletrônico $A^{1} \Pi$, e $w^{\prime \prime}$. $=1285,08 \mathrm{~cm}^{-1}$ e $w^{\prime \prime}{ }_{\mathrm{c}} x^{\prime \prime}{ }_{\mathrm{e}}=6,46 \mathrm{~cm}^{-1}$, para o estado eletrónico fundamental $X^{1} \Sigma$ (Huber e Herzberg, 1950). 
TABELA 2. Transições puramente vibracionais do ${ }^{12} \mathrm{C}^{32} \mathrm{~S}$

\begin{tabular}{|c|c|c|c|c|}
\hline $\begin{array}{l}\text { BANDA } \\
\text { VIBRAC. } \\
\text { [v',v'] }\end{array}$ & $\begin{array}{c}\text { COMPRIM. } \\
\text { DE ONDA } \\
\lambda \\
{[\mathbf{A}]}\end{array}$ & $\begin{array}{c}\text { FREQÜÊNCIA } \\
\mathbf{v} \\
{[\mathbf{H z}]}\end{array}$ & $\begin{array}{c}\text { TAXA EMIS. } \\
\text { ESPONT. } \\
\text { A }_{\mathbf{v}^{\prime}, \mathbf{v}^{\prime \prime}} \\
{\left[\mathbf{s}^{-1}\right]}\end{array}$ & $\begin{array}{c}\text { COEFIC. DE } \\
\text { ABSORÇÃO } \\
\text { B }_{v^{\prime}, v^{\prime \prime}} \\
{\left[\mathrm{cm}^{2} \mathbf{e r g}^{-1} \mathbf{s}^{-1}\right]}\end{array}$ \\
\hline$(1,0)$ & 7,861 & $38,138 \times 10^{12}$ & $6,600 \times 10^{0}$ & $6,420 \times 10^{5}$ \\
\hline$(2,1)$ & 7,941 & $37,751 \times 10^{12}$ & $1,280 \times 10^{1}$ & $1,284 \times 10^{6}$ \\
\hline$(3,2)$ & 8,024 & $37,364 \times 10^{12}$ & $1,862 \times 10^{1}$ & $1,926 \times 10^{6}$ \\
\hline$(4,3)$ & 8,108 & $36,976 \times 10^{12}$ & $2,406 \times 10^{1}$ & $2,568 \times 10^{6}$ \\
\hline$(5,4)$ & 8,193 & $36,589 \times 10^{12}$ & $2,914 \times 10^{1}$ & $3,210 \times 10^{6}$ \\
\hline$(5,3)$ & 4,074 & $73,565 \times 10^{12}$ & $1,190 \times 10^{0}$ & $1,614 \times 10^{4}$ \\
\hline$(4,2)$ & 4,033 & $74,340 \times 10^{12}$ & $7,371 \times 10^{-1}$ & $9,682 \times 10^{3}$ \\
\hline$(5,2)$ & 2,703 & $110,929 \times 10^{12}$ & $2,736 \times 10^{-2}$ & $1,082 \times 10^{2}$ \\
\hline$(2,0)$ & 3,950 & $75,889 \times 10^{12}$ & $1,307 \times 10^{-1}$ & $1,614 \times 10^{3}$ \\
\hline$(3,0)$ & 2,647 & $113,253 \times 10^{12}$ & $2,911 \times 10^{-3}$ & $1,082 \times 10^{1}$ \\
\hline$(4,0)$ & 1,996 & $150,230 \times 10^{12}$ & $7,686 \times 10^{-5}$ & $1,223 \times 10^{-1}$ \\
\hline$(5,0)$ & 1,605 & $186,819 \times 10^{12}$ & $2,378 \times 10^{-6}$ & $1,968 \times 10^{-3}$ \\
\hline$(3,1)$ & 3,991 & $75,115 \times 10^{12}$ & $3,802 \times 10^{1}$ & $4,841 \times 10^{3}$ \\
\hline$(4,1)$ & 2,674 & $112,091 \times 10^{12}$ & $1,129 \times 10^{2}$ & $4,326 \times 10^{1}$ \\
\hline$(5,1)$ & 2,016 & $148,680 \times 10^{12}$ & $3,725 \times 10^{-4}$ & $6,117 \times 10^{-1}$ \\
\hline
\end{tabular}

O sistema de equações que descrevemos em (8) e (9) foi solucionado com o auxílio de um Programa FORTRAN IV de inversão de matrizes em que substituímos, de modo adequado, uma de suas equações por aquela que normaliza todo o sistema. Com o objetivo de comparar nossos resultados com os da Literatura, nós deduzimos as populações para 3 distâncias heliocêntricas: $0,5,0,71$ e 1,0 UA. Os resultados aparecem mostrados na Tabela 3.

Com o auxíiio da expressão (Herzberg, 1950)

$$
I_{w}=n_{v} h v_{w}-A w
$$

na qual $n_{v^{\prime}}$ é a fração da população vibracional no nível superior $v^{\prime}, e v_{v^{\prime}}$ é a freqüência $(e m \mathrm{~Hz}) d a$ transição considerada, nós obtivemos as intensidades de 9 bandas vibracionais do Sistema (A-X) da molécula CS. Os resultados foram normalizados pelo valor relativo à banda $(0,0)$ e aparecem mostrados na Tabela 4. 


\begin{tabular}{|c|c|c|}
\hline$r=0,50 \cup A$ & $r=0,71 \cup A$ & $r=1,00 \cup A$ \\
\hline & $X^{1} \Sigma^{+}$ & \\
\hline$n_{0}^{\prime \prime}=9,9 \times 10^{-1}$ & $n_{0}^{\prime \prime}=9,9 \times 10^{-1}$ & $n_{0}^{\prime \prime}=9,9 \times 10^{-1}$ \\
\hline$n^{\prime \prime}{ }_{1}=3,7 \times 10^{-4}$ & $n^{\prime \prime}{ }_{1}=1,8 \times 10^{-4}$ & $\mathrm{n}^{\prime \prime}{ }_{1}=9,3 \times 10^{-5}$ \\
\hline$n_{z}^{n}=1,9 \times 10^{-6}$ & $n^{\prime \prime}{ }_{2}=8,5 \times 10^{6}$ & $n_{2}^{\prime \prime}=4,1 \times 10^{-6}$ \\
\hline$n n_{3}=1,9 \times 10^{-8}$ & $\mathrm{n}_{3}=9,6 \times 10^{-7}$ & $\mathrm{n}_{3}=4,8 \times 10^{-7}$ \\
\hline$n^{n}{ }_{4}=2,5 \times 10^{-7}$ & $\mathrm{n}_{4}=1,2 \times 10^{-7}$ & $\mathrm{n}_{4}=6,2 \times 10^{-8}$ \\
\hline \multirow[t]{2}{*}{$n^{\prime \prime}{ }_{5}=6,8 \times 10^{-7}$} & $\mathrm{n}^{\prime \prime}{ }_{5}=3,4 \times 10^{-7}$ & $n^{\prime \prime}=1,7 \times 10^{7}$ \\
\hline & $A^{1} \Pi$ & \\
\hline $\mathrm{n}_{\mathrm{B}}^{1}=2,9 \times 10^{-10}$ & $n_{B}^{\prime}=1,4 \times 10^{-10}$ & $n_{6}^{\prime}=7,3 \times 10^{-11}$ \\
\hline$n_{7}^{\prime}=3,6 \times 10^{-11}$ & $n_{3}^{\prime}=1,8 \times 10^{-11}$ & $\mathbf{n}_{7}^{\prime}=9,1 \times 10^{-12}$ \\
\hline$n_{g}^{\prime}=5,4 \times 10^{-12}$ & $n_{8}^{\prime}=2,7 \times 10^{-12}$ & $n_{8}^{\prime}=1,3 \times 10^{12}$ \\
\hline$n_{9}^{\prime}=3,3 \times 10^{-13}$ & $n_{9}^{\prime}=1,6 \times 10^{-13}$ & $\mathrm{n}_{9}^{1}=8,2 \times 10^{-14}$ \\
\hline$n^{\prime}{ }^{\prime}=1,8 \times 10^{-14}$ & $n_{-10}^{\prime}=8,4 \times 10^{-15}$ & $\mathrm{n}_{10}=4,1 \times 10^{-15}$ \\
\hline$n^{\prime}{ }_{11}=1,1 \times 10^{-15}$ & $n^{\prime}{ }_{11}=4,4 \times 10^{-16}$ & $n^{\prime \prime}=2,0 \times 10^{.16}$ \\
\hline
\end{tabular}

TABELA 4. Intensidades das bandas vibracionais do ${ }^{12} \mathrm{C}^{32} \mathrm{~S}$

\begin{tabular}{|c|c|c|c|c|c|}
\hline $\begin{array}{l}\text { BANDA } \\
\text { VIBRACIONAL } \\
\left(\mathbf{v}^{\prime}, \mathbf{v}^{\prime \prime}\right)\end{array}$ & $\begin{array}{l}\text { DISTÂNC } \\
0,50\end{array}$ & $\begin{array}{l}\text { IELIOCEN } \\
\text { r (UA) } \\
\mathbf{0 , 7 1}\end{array}$ & $\begin{array}{l}\text { CA } \\
\qquad 1,00\end{array}$ & $\begin{array}{l}\text { INTENSIDADES } \\
\text { (Dados Observacionais) }\end{array}$ & $\begin{array}{l}\text { INTENSIDADES } \\
\text { (Literatura) }\end{array}$ \\
\hline$(0,0)$ & $1,0 \times 10^{0}$ & $1,0 \times 10^{0}$ & $1,0 \times 10^{0}$ & $1,0 \times 10^{0(a)} \quad 1,0 \times 10^{0(b)}$ & $1,0 \times 10^{0}(c)$ \\
\hline$(1,0)$ & $3,0 \times 10^{-2}$ & $3,0 \times 10^{-2}$ & $3,0 \times 10^{-2}$ & $5,0 \times 10^{-2}$ & $4,0 \times 10^{-2(c)}$ \\
\hline$(0,1)$ & $1,3 \times 10^{-1}$ & $1,3 \times 10^{-1}$ & $1,3 \times 10^{-1}$ & $1,2 \times 10^{-1(a)}$ & $1,7 \times 10^{-1(c)}$ \\
\hline$(1,1)$ & $7,0 \times 10^{-2}$ & $8,0 \times 10^{-2}$ & $7.0 \times 10^{-2}$ & $8,0 \times 10^{-2(8)}$ & $6,0 \times 10^{2(0)}$ \\
\hline$(0,2)$ & $1,0 \times 10^{-2}$ & $1,0 \times 10^{-2}$ & $1,0 \times 10^{-2}$ & $1,0 \times 10^{-2(a)}$ & $2,0 \times 10^{-2(c)}$ \\
\hline$(1,2)$ & $3,0 \times 10^{-2}$ & $3,0 \times 10^{-2}$ & $3,0 \times 10^{-2}$ & $1,2 \times 10^{-2(a)}$ & $3,0 \times 10^{-2(0)}$ \\
\hline$(2,1)$ & $1,0 \times 10^{2}$ & $1,0 \times 10^{-2}$ & $1,0 \times 10^{-2}$ & & \\
\hline$(2,2)$ & $5,0 \times 10^{-3}$ & $6,0 \times 10^{-3}$ & $5,0 \times 10^{-3}$ & & \\
\hline$(2,3)$ & $4,0 \times 10^{-3}$ & $5,0 \times 10^{-3}$ & $4,0 \times 10^{-3}$ & & \\
\hline
\end{tabular}

(a) Obsenaçōes realizadas por Smith et al (1980), para $r=0,52$ UA.

(b) Observaçōes realizadas por Feldman et al (1980), para $r=0,71$ UA.

(c) Resultados teóricos de Krishna Swamy (1981) 


\section{COMENTÁRIOS E DISCUSSÃO DOS RESULTADOS}

O monossulfeto de carbono $\left({ }^{12} \mathrm{C}^{32} \mathrm{~S}\right)$ é uma espécie molecular que aparece em diversos meios astrofísicos. No Meio Interestelar eíe faz a função de um importante traçador das condições físicas nas nuvens moleculares densas que são regiões de formação de estrelas. Em Cometas, sua emissão no ultravioleta foi inicialmente observada por Smith et al. (1980), na coma do Cometa West enquanto que sua taxa de produção em 15 cometas, para a distância heliocêntrica de 1,0 UA, varia entre $10^{24}-10^{25}$ moléculas/s (Sanzovo et ai., 1993). Como a escala de tempo característica de colisões entre átomos neutros ou íons nas comas cometárias $\left(\sim 10^{7} \mathrm{~s}\right)$ é muito superior àquela relativa ao processo de absorção da radiação solar na região visível, para espécies como $\mathrm{C}_{2}$ e CN ( 10-100 s) quando o cometa se encontra a 1,0 UA de distância do Sol, conclui-se que a absorção da radiação soiar é o principal mecanismo de excitação. Neste trabalho nós aplicamos o mecanismo de excitação por fluorescência ressonante solar para a espécie molecular ${ }^{12} \mathrm{C}^{32} \mathrm{~S}$ nos Cometas West (1976VI) e P/ Bradfield $(1979 X)$ e obtivemos as populações fracionais e as intensidades das linhas de emissão da molécula. Além de permitir a determinação das populações relativas a 12 bandas vibracionais da molécula para o sistema eletrônico $A^{1} \Pi-X^{\prime} \Sigma^{+}$, o mecanismo de fluorescência ressonante solar mostrou-se, também, eficaz para explicar as fortes emissões do monossulfeto de carbono nestes cometas. As intensidades de 9 bandas vibracionais foram deduzidas e os resultados, apresentados na Tabela 4, parecem reproduzir melhor os dados observacionais que aquele obtidos teoricamente por Krishna Swamy (1981). A diferença entre seus valores o aqueles obtidos neste trabalho pode ser explicada, muito provavelmente, nos valores mais precisos das origens de bandas vibracionais, aqui utilizados, para a referida molécula.

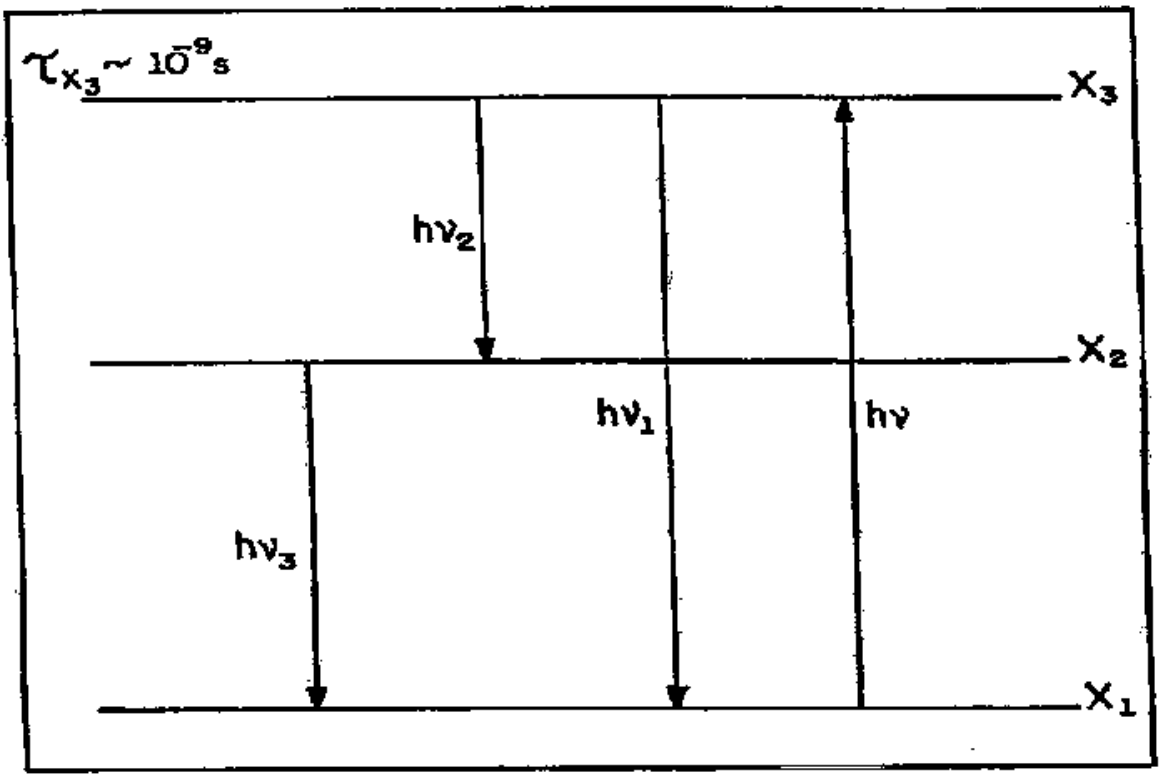

Figura 1 - O Mecanismo de Fluorescência Ressonante 
CANAVES, M. V.; SANZOVO, G. C. Comets: Populations and Intensities of Nine Vibrational Bands of Carbon Monosulfide ( $\left.{ }^{12} \mathrm{C}^{32} \mathrm{~S}\right)$. Semina:Ci. Exatas/Tecnol., v. 17, n. 4, p. 369-376, Dec. 1996.

ABSTRACT: A theoretical study of resonance fluorescence mechanism for carbon monosulfide $\left({ }^{12} C^{32} S\right)$ in the comae of Comets West (1976VI) and P/Bradfield (1979X) using the solar radiation field is presented. We obtained the relative populations in several different vibrational levels, and the intensities of 9 vibrational bands of CS molecule.

KEY WORDS: Comets; resonance fluorescence process; CS: vibrational populations; CS: intensities of vibrational bands.

\section{REFERÊNCIAS BIBLIOGRÁFICAS}

ALLEN, C. W. Astrophysical Quantities, $3^{\text {re }}$ ed. London : Athlone Press, 1973.

AVERSEN, J. C., GRIFFIN, R. N., PEARSON JR, B. D. Determination of extraterrestrial solar spectral irradiance from a research aircraft. Appl. Optics (USA), v. 8, n. 11, p. 2215-32, 1969

BROADFOOT, A. L. Solar spectrum 2100-3200A. Astrophys $J$, v. 173, n. 3, p. $681-\&, 1972$.

COXON, J. A., MARCOUX, P. J., SETSER, D. W. Intensity and wavelength measurements for CS+(A2PI-X2SIGMA) and CS(A1PI-X1SIGMA+) band systems excited by reaction of metastable he (2S-3) with CS2. Chem Phys, v. 17, n. 4, p. 403-415, 1976.

HERZBERG, G. Spectra of diatomic molecules. New York : van Nostrand Reinhold, 1950.

HUBER, R. P., HERZBERG, G. Molecular spectra and molecular structure. IV.Constants of diatomic molocuies. New York: van Nostrand Reinhold, 1979.
LABS, D., NECKEL, $H$. The radiation of the solar pholosphere from 2000 a to $100 \mu$. $Z$. Astrophys (Gemany), v. 69, n. 1, p. $1-73,1968$

LARSSON, M. Birth and infancy of stars. In: LUCAS, R., OMONT, A., STORA, R. (Ed.). Les houches 1982, Session XLI. New York: North-Holland, 1983.

LÜST, R. Chemistry in comets. Top Curr Chem, v. 99: p. 73-98, 1981.

PENNER, S. S. Quantitative molecular spectroscopy and gas emissivities. London: Addison-Wesley, 1959.

SANZOVO, G. C., SINGH, P. D., HUEBNER, W. F. CS band intensity and column densities and production-rates of 15 comets. Astron $J$, v. 106, n. 3, p. 1237-1240, 1993.

SMITH, A. M., STECHER, T. P., CASSWELL, L. Production of carbon, sulfur and CS in comet west. Astrophys $J, v .242$, ก. 1, p. 402-\&, 1980.

SWINGS, P. Lick Obs Bull, v. 19, p. 131, 1941. 